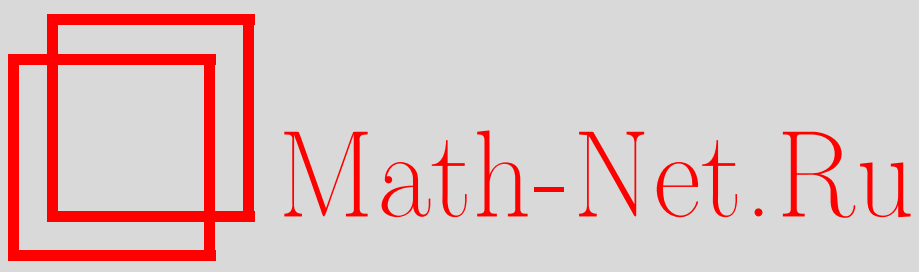

A. M. Khludnev, T. S. Popova, The junction problem for two weakly curved inclusions in an elastic body, Sibirsk. Mat. Zh., 2020, Volume 61, Number 4, 932-945

DOI: https://doi.org/10.33048/smzh.2020.61.414

Use of the all-Russian mathematical portal Math-Net.Ru implies that you have read and agreed to these terms of use http://www . mathnet.ru/eng/agreement

Download details:

IP: 54.84 .234 .179

April 26, 2023, 13:38:54

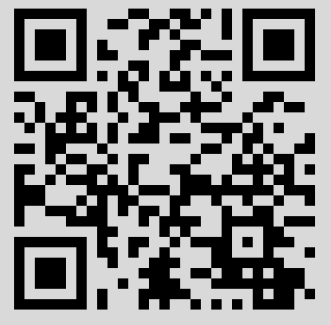


Сибирский математический журнал Июль-август, 2020. Том 61, № 4

УДК 517.958

\title{
О ЗАДАЧЕ СОПРЯЖЕНИЯ ДВУХ СЛАБО ИСКРИВЛЕННЫХ ВКЛЮЧЕНИЙ В УПРУГОМ ТЕЛЕ
}

\section{А. М. Хлуднев, Т. С. Попова}

\begin{abstract}
Аннотация. Рассматриваются краевые задачи, описывающие равновесие двумерных упругих тел с тонкими слабо искривленными включениями при наличии отслоения. Наличие отслоения означает существование трещины между включениями и упругим телом. На берегах трещины задаются нелинейные краевые условия в виде неравенств, исключающие взаимное проникание берегов, что приводит к формулировке проблем в виде задач с неизвестной областью контакта. Предполагается, что включения имеют точку взаимного контакта. Найдены условия сопряжения в контактной точке. Исследован предельный переход к бесконечности по параметру жесткости одного из тонких включений. В частности, построены предельные модели и проведен анализ их свойств.
\end{abstract}

DOI 10.33048/smzh.2020.61.414

Ключевые слова: краевая задача, упругое тело, включение, трещина, условия сопряжения.

1. Введение. В работе исследуются краевые задачи сопряжения двух слабо искривленных включений, расположенных в упругом теле, при наличии трещин отслоения. На берегах трещин при этом задаются нелинейные краевые условия в виде неравенств, не допускающие взаимного проникания противоположных берегов. Целью данной работы являются отыскание краевых условий в точке сопряжения и доказательство разрешимости соответствующих краевых задач. Рассматриваются сопряжение двух упругих включений, а также сопряжение упругого включения с жестким.

В последние годы проведены обширные исследования краевых задач равновесия упругих и неупругих тел с трещинами в рамках моделей с указанными нелинейными краевыми условиями на берегах [1-7]. Аналогичные результаты получены и в случае задач равновесия упругих тел с тонкими включениями при наличии трещин отслоения [8-14]. Из работ, относящихся к тематике сопряжения, отметим исследования [15-22], где рассматривались задачи сопряжения упругих объектов друг с другом. Задачи сопряжения упругих, жестких и полужестких включений, расположенных в упругих телах, при наличии отслоений с нелинейными краевыми условиями на берегах трещин можно найти в [2328]. При этом для описания тонких упругих включений использовались модели балок Бернулли - Эйлера и Тимошенко.

Работа выполнена при поддержке Математического центра в Академгородке.

(c) 2020 Хлуднев А. М., Попова Т. С. 
В случае слабо искривленных включений, рассмотренных в данной работе, возникает целый ряд особенностей, обусловленных ненулевой кривизной включений. В частности, структура поля перемещений для жестких слабо искривленных включений заметно сложнее, чем для прямолинейных включений. Рассматриваются два возможных варианта взаимного контакта включений. Первый вариант соответствует излому составного включения в точке контакта, а второй вариант характеризуется отсутствием излома, что соответствует сохранению угла между включениями в указанной точке в процессе деформирования.

2. Формулировка задачи равновесия. Случай с изломом. Сформулируем задачу равновесия упругого тела с двумя тонкими упругими слабо искривленными включениями. Пусть $\Omega \subset \mathbb{R}^{2}-$ ограниченная область с гладкой границей $\Gamma ; \gamma=\gamma_{1} \cup \gamma_{2} \cup\{(0,0)\}, \Omega_{\gamma}=\Omega \backslash \bar{\gamma}$, где

$$
\gamma_{i}=\left\{\left(x_{1}, x_{2}\right) \mid x_{2}=\varphi\left(x_{1}\right), x_{1} \in s_{i}\right\}, \quad s_{1}=(-1,0), s_{2}=(0,1),
$$

$\varphi:(-1,1) \rightarrow \mathbb{R}-$ заданная функция такая, что $\varphi(0)=0$, обладающая следующей гладкостью:

$$
\varphi \in H^{1}\left(s_{i}\right), \quad \varphi_{, 11} \in L^{\infty}\left(s_{i}\right), \quad i=1,2 .
$$

Считаем, что срединные линии тонких упругих включений совпадают с $\gamma_{i}$. Таким образом, включения имеют точку контакта $(0,0)$. Упругое тело при этом занимает область $\Omega_{\gamma}$. Будем предполагать, что в точке $(0,0)$ имеется излом включения $\gamma$. Термин «излом» означает, что мы имеем фактически два включения $\gamma_{1}$ и $\gamma_{2}$, не связанные между собой в точке $(0,0)$. Говоря другими словами, если убрать окружающее упругое тело, то два включения $\gamma_{1}, \gamma_{2}$ можно перемещать независимо друг от друга.

Для описания слабо искривленных включений используется модель Бернулли - Эйлера. По поводу подходящих моделей упругих пологих оболочек (и соответствующих одномерных слабо искривленных стержней) см., например, $\left[29\right.$, гл. 1]. Пусть $\nu=\left(\nu_{1}, \nu_{2}\right)-$ единичный вектор нормали к $\gamma$, а $\tau=\left(\nu_{2},-\nu_{1}\right)$; $B=\left\{b_{i j k l}\right\}, i, j, k, l=1,2$, - заданный положительно определенный тензор коэффициентов упругости:

$$
\begin{gathered}
b_{i j k l}=b_{j i k l}=b_{k l i j}, i, j, k, l=1,2 ; \quad b_{i j k l} \in L^{\infty}(\Omega), \\
b_{i j k l} \varphi_{i j} \varphi_{k l} \geq c_{0}|\varphi|^{2} \quad \forall \varphi_{j i}=\varphi_{i j}, \quad c_{0}=\mathrm{const}>0 .
\end{gathered}
$$

По повторяющимся индексам производится суммирование. Все величины с двумя нижними индексами в дальнейшем считаются симметричными по этим индексам; $f=\left(f_{1}, f_{2}\right) \in L^{2}(\Omega)^{2}$ - заданный вектор внешних сил, действующих на упругое тело, $k \in L^{\infty}\left(s_{i}\right)$ - заданная кривизна срединных линий тонких включений, равная $\varphi_{, 11}\left(1+(\varphi, 1)^{2}\right)^{-3 / 2}$.

Предположим, что положительный (по отношению к нормали $\nu$ ) берег включения $\gamma$ отслаивается, образуя тем самым трещину между упругим телом и включением. На берегах трещины будем задавать краевые условия в виде неравенств, обеспечивающие взаимное непроникание берегов. Для упрощения формулировки краевых условий сопряжения в точке $(0,0)$ будем считать, что $\varphi, 1(0+)=0$. Это предположение не снижает общности результата, поскольку всегда можно выбрать систему координат так, что $\varphi_{, 1}(0+)=0$. 
Постановка задачи равновесия упругого тела с включениями $\gamma_{1}, \gamma_{2}$ состоит в следующем. Требуется найти вектор перемещений $u=\left(u_{1}, u_{2}\right)$ и тензор напряжений $\sigma=\left\{\sigma_{i j}\right\}, i, j=1,2$, определенные в $\Omega_{\gamma}$, и перемещения точек тонких включений $v, w$, определенные в $s_{1} \cup s_{2}$, такие, что

$$
\begin{gathered}
-\operatorname{div} \sigma=f, \quad \sigma-B \varepsilon(u)=0 \text { в } \Omega_{\gamma}, \\
v_{, 1111}+k\left(w_{, 1}+k v\right)=\left[\sigma_{\nu}\right] p \text { на } s_{i}, \quad i=1,2, \\
-w_{, 11}-(k v)_{, 1}=\left[\sigma_{\tau}\right] p \text { на } s_{i}, \quad i=1,2, \\
u=0 \text { на } \Gamma ; \quad v_{, 11}=v_{, 111}=w_{, 1}+k v=0, \quad x_{1}=-1,1, \\
{\left[u_{\nu}\right] \geq 0, \quad v=u_{\nu}^{-}, \quad w=u_{\tau}^{-} \quad \text { на } \gamma,} \\
\sigma_{\nu}^{+} \leq 0, \sigma_{\tau}^{+}=0, \sigma_{\nu}^{+}\left[u_{\nu}\right]=0 \quad \text { на } \gamma, \\
{[v(0)]_{p}=[w(0)]_{p}=\left[v_{, 111}(0)\right]^{p}=\left[\left(w_{, 1}+k v\right)(0)\right]^{p}=0, \quad v_{, 11}(0 \pm)=0 .}
\end{gathered}
$$

Здесь $[h]=h^{+}-h^{-}-$скачок функции $h$ на $\gamma$, где $h^{ \pm}-$значения функции $h$ на положительном и отрицательном берегах разреза $\gamma$ в соответствии с выбранным направлением нормали $\nu ; \varepsilon(u)=\left\{\varepsilon_{i j}(u)\right\}$ - тензор деформаций, $\varepsilon_{i j}(u)=\frac{1}{2}\left(u_{i, j}+\right.$ $\left.u_{j, i}\right), i, j=1,2 ; \sigma \nu=\left(\sigma_{1 j} \nu_{j}, \sigma_{2 j} \nu_{j}\right), \sigma_{\nu}=\sigma_{i j} \nu_{j} \nu_{i}, \sigma_{\tau}=\sigma_{i j} \nu_{j} \tau_{i}, u_{\nu}=u \nu, u_{\tau}=u \tau$, $p=\sqrt{1+\varphi_{, 1}^{2}}$.

Скачки $[\cdot]_{p}$ и $[\cdot]^{p}$ функций, заданных на оси $x_{1}$, в точке $x_{1}=0$ определяются следующими формулами:

$$
[m(0)]_{p}=m(0+)-\left(p^{-1} m\right)(0-) ; \quad[m(0)]^{p}=m(0+)-(p m)(0-) .
$$

Второе и третье равенства (2.5) следует понимать так: $v\left(x_{1}\right)=u_{\nu}^{-}\left(x_{1}, \varphi\left(x_{1}\right)\right)$, $x_{1} \in s_{i}, i=1,2$. При этом (2.1) - уравнения равновесия упругого тела и уравнение состояния (закон Гука), а (2.2), (2.3) представляют уравнения равновесия тонких слабо искривленных включений. Правые части уравнений равновесия тонких включений $(2.2),(2.3)$ описывают силы, действующие на включения со стороны упругого тела. Первое краевое условие из (2.5) обеспечивает взаимное непроникание берегов трещины, а второе и третье условия гарантируют равенство перемещений точек упругого тела и тонкого включения на $\gamma^{-}$. Вторая группа краевых условий (2.4) соответствует нулевому моменту, нулевой перерезывающей силе и нулевой деформации растяжения (сжатия). Что касается краевых условий (2.6), то они типичны при формулировке краевых задач теории трещин с неизвестной областью контакта (см. [1]). В частности, если в заданной точке $x_{0}$ контакт отсутствует, т. е. $\left[u_{\nu}\left(x_{0}\right)\right]>0$, получаем нулевое значение поверхностной силы: $(\sigma \nu)^{+}\left(x_{0}\right)=0$. С другой стороны, если поверхностная сила ненулевая, т. е. $\sigma_{\nu}^{+}\left(x_{0}\right)<0$, то имеем условие контакта $\left[u_{\nu}\left(x_{0}\right)\right]=0$. Краевые условия (2.7) описывают условия сопряжения двух включений в точке $(0,0)$. В частности, изгибающие моменты равны нулю; скачки $[\cdot]_{p}$ для перемещений тонких включений равны нулю; скачки [·. ${ }^{p}$ перерезающих сил и сил, действующих в касательном направлении, также равны нулю. Поскольку в точке $(0,0)$ имеется излом между включениями, скачок функции $v_{, 1}$ в точке 0 , вообще говоря, ненулевой.

Как будет установлено ниже, соотношения (2.1)-(2.7) в точности эквивалентны вариационной формулировке задачи минимизации функционала энергии на подходящем множестве функций. При этом функционал энергии содержит слагаемые, соответствующие энергии деформирования упругого тела, работе внешних сил, энергии изгиба и растяжения тонкого включения. Приведем 
вариационную формулировку задачи (2.1)-(2.7). Введем для этого множество допустимых перемещений

$$
\begin{aligned}
K=\left\{(u, v, w) \mid u \in H_{\Gamma}^{1}\left(\Omega_{\gamma}\right)^{2}, v \in H^{2}\left(s_{i}\right), w\right. & \in H^{1}\left(s_{i}\right), i=1,2, \\
& {\left.\left[u_{\nu}\right] \geq 0, v=u_{\nu}^{-}, w=u_{\tau}^{-} \text {на } \gamma\right\}, }
\end{aligned}
$$

где пространство Соболева $H_{\Gamma}^{1}\left(\Omega_{\gamma}\right)$ определяется следующим образом:

$$
H_{\Gamma}^{1}\left(\Omega_{\gamma}\right)=\left\{\phi \in H^{1}\left(\Omega_{\gamma}\right) \mid \phi=0 \text { на } \Gamma\right\} .
$$

Рассмотрим функционал энергии

$$
\pi(u, v, w)=\frac{1}{2} \int_{\Omega_{\gamma}} \sigma(u) \varepsilon(u)-\int_{\Omega_{\gamma}} f u+\frac{1}{2} \sum_{i=1}^{2} \int_{s_{i}} v_{, 11}^{2}+\frac{1}{2} \sum_{i=1}^{2} \int_{s_{i}}\left(w_{, 1}+k v\right)^{2} .
$$

Здесь и далее для краткости $\sigma_{i j}(u) \varepsilon_{i j}(u)$ обозначается через $\sigma(u) \varepsilon(u)$. Тогда задача минимизации:

$$
\text { найти }(u, v, w) \in K, \text { так что } \pi(u, v, w)=\inf _{K} \pi,
$$

имеет решение, удовлетворяющее вариационному неравенству

$$
\begin{gathered}
(u, v, w) \in K \\
\int_{\Omega_{\gamma}} \sigma(u) \varepsilon(\bar{u}-u)-\int_{\Omega_{\gamma}} f(\bar{u}-u)+\sum_{i=1}^{2} \int_{s_{i}} v_{, 11}\left(\bar{v}_{, 11}-v_{, 11}\right) \\
+\sum_{i=1}^{2} \int_{s_{i}}\left\{\left(w_{, 1}+k v\right)\left(\bar{w}_{, 1}+k \bar{v}-w_{, 1}-k v\right)\right\} \geq 0 \quad \forall(\bar{u}, \bar{v}, \bar{w}) \in K .
\end{gathered}
$$

Разрешимость задачи (2.8), (2.9) установлена в [11], поэтому соответствующие рассуждения приводить не будем. Отметим также, что решение единственно.

Можно доказать, что на классе гладких решений задачи $(2.8),(2.9)$ и (2.1)(2.7) эквиваленты. Это означает, что все соотношения (2.1)-(2.7) вытекают из $(2.8),(2.9)$ и, наоборот, неравенство $(2.8),(2.9)$ можно вывести из $(2.1)-(2.7)$.

Предложение 1. Задачи (2.8), (2.9) и (2.1)-(2.7) эквивалентны на классе достаточно гладких решений.

ДокАЗАтЕльство. Пусть имеет место (2.8), (2.9). Сначала с помощью соответствующей подстановки в (2.9) стандартным образом убеждаемся, что выполнено уравнение равновесия из (2.1). Краевые условия (2.6) типичны для контактных задач данного вида, и их вывод опустим (см. [1,11]). Далее выберем в (2.9) пробные функции вида $(\bar{u}, \bar{v}, \bar{w})=(u, v, w) \pm(\tilde{u}, \tilde{v}, \widetilde{w}),(\tilde{u}, \tilde{v}, \widetilde{w}) \in K,\left[\tilde{u}_{\nu}\right]=$ 0 на $\gamma$. Получим

$$
\int_{\Omega_{\gamma}} \sigma(u) \varepsilon(\tilde{u})-\int_{\Omega_{\gamma}} f \tilde{u}+\sum_{i=1}^{2} \int_{s_{i}} v_{, 11} \tilde{v}_{, 11}+\sum_{i=1}^{2} \int_{s_{i}}\left(w_{, 1}+k v\right)\left(\widetilde{w}_{, 1}+k \tilde{v}\right)=0,
$$


следовательно, интегрируя по частям, находим

$$
\begin{aligned}
-\int_{\gamma}\left[\sigma_{\nu}\right] \tilde{u}_{\nu} & -\int_{\gamma}\left[\sigma_{\tau} \tilde{u}_{\tau}\right]+\sum_{i=1}^{2} \int_{s_{i}} v_{, 1111} \tilde{v} \\
& -\sum_{i=1}^{2} \int_{s_{i}}\left(w_{, 1}+k v\right)_{, 1} \tilde{w}+\sum_{i=1}^{2} \int_{s_{i}}\left(w_{, 1}+k v\right) k \tilde{v}+\left.v_{, 11} \tilde{v}_{, 1}\right|_{-1} ^{0} \\
+ & \left.v_{, 11} \tilde{v}_{, 1}\right|_{0} ^{1}-\left.v_{, 111} \tilde{v}\right|_{-1} ^{0}-\left.v_{, 111} \tilde{v}\right|_{0} ^{1}+\left.\left(w_{, 1}+k v\right) \tilde{w}\right|_{-1} ^{0}+\left.\left(w_{, 1}+k v\right) \tilde{w}\right|_{0} ^{1}=0 .
\end{aligned}
$$

Временно считаем, что в (2.10) тестовые функции удовлетворяют условиям $\tilde{v}=$ $\tilde{v}_{, 1}=\widetilde{w}=0$ при $x_{1}=-1,0,1$. Равенство нулю производной $\tilde{v}_{, 1}$ при $x_{1}=0$ означает, что $\tilde{v}_{, 1}(0 \pm)=0$. Получим тождество

$-\int_{\gamma}\left[\sigma_{\nu}\right] \tilde{u}_{\nu}-\int_{\gamma}\left[\sigma_{\tau} \tilde{u}_{\tau}\right]+\sum_{i=1}^{2} \int_{s_{i}} v_{, 1111} \tilde{v}-\sum_{i=1}^{2} \int_{s_{i}}\left(w_{, 1}+k v\right)_{, 1} \tilde{w}+\sum_{i=1}^{2} \int_{s_{i}}\left(w_{, 1}+k v\right) k \tilde{v}=0$,

справедливое для всех выбранных функций. Отсюда вытекают уравнения равновесия $(2.2),(2.3)$, так как $d \gamma=p d x_{1}$. Вернемся к тождеству $(2.10)$. В силу имеющейся гладкости решения и второго и третьего краевых условий (2.5) имеем $[v(0)]_{p}=[w(0)]_{p}=0$. Действительно, кривая $\gamma$ липшицева. Поскольку $u \in$ $H^{1}\left(\Omega_{\gamma}\right)^{2}$, то $\left.u\right|_{\gamma^{-}} \in H^{1 / 2}(\gamma)$. Поэтому в силу краевых условий $v=u_{\nu}^{-}, w=u_{\tau}^{-}$на $\gamma$ и имеющейся гладкости функций $v, w$ перемещения включений в точке $(0,0)$ обязаны совпадать. Функции $v, w$ суть перемещения тонких включений вдоль нормали и касательного направления. Таким образом, склейка перемещений в точке $(0,0)$ приводит в краевым условиям $[v(0)]_{p}=[w(0)]_{p}=0$. Здесь принимаем во внимание сделанное предположение $\varphi, 1(0+)=0$. Следует также иметь в виду, что скачки $[\cdot]_{p}$ тестовых функций $\tilde{v}, \widetilde{w}$ при $x_{1}=0$ нулевые. Поэтому из (2.10) получаем вторую группу краевых условий (2.4) и, кроме того,

$$
\left(v_{, 11} \tilde{v}_{, 1}\right)(0-)-\left(v_{, 11} \tilde{v}_{, 1}\right)(0+)+\left[v_{, 111}(0)\right]^{p} \tilde{v}(0+)-\left[\left(w_{, 1}+k v\right)(0)\right]^{p} \widetilde{w}(0+)=0 .
$$

Отсюда следуют оставшиеся краевые условия из (2.7). Таким образом, из (2.8), (2.9) получаем все соотношения (2.1)-(2.7).

Обратно, пусть выполнено (2.1)-(2.7). Умножим первое уравнение (2.1) на $\bar{u}-u$, а уравнения $(2.2),(2.3)-$ на $\bar{v}-v, \bar{w}-w$ соответственно, проинтегрируем по $\Omega_{\gamma}, s_{i}$. После интегрирования по частям получим

$$
\begin{aligned}
& \int_{\gamma}[\sigma \nu(\bar{u}-u)]+\int_{\Omega_{\gamma}} \sigma(u) \varepsilon(\bar{u}-u)-\int_{\Omega_{\gamma}} f(\bar{u}-u)+\sum_{i=1}^{2} \int_{s_{i}} v_{, 11}\left(\tilde{v}_{, 11}-v_{, 11}\right) \\
& \quad+\sum_{i=1}^{2} \int_{s_{i}}\left(w_{, 1}+k v\right)\left(\bar{w}_{, 1}+k \bar{v}-w_{, 1}-k v\right)-\sum_{i=1}^{2} \int_{s_{i}}\left[\sigma_{\tau}\right] p(\bar{w}-w) \\
& -\sum_{i=1}^{2} \int_{s_{i}}\left[\sigma_{\nu}\right] p(\bar{v}-v)-\left.v_{, 11}\left(\bar{v}_{, 1}-v_{, 1}\right)\right|_{-1} ^{0}-\left.v_{, 11}\left(\bar{v}_{, 1}-v_{, 1}\right)\right|_{0} ^{1}+\left.v_{, 111}(\bar{v}-v)\right|_{-1} ^{0} \\
& \quad+\left.v_{, 111}(\bar{v}-v)\right|_{0} ^{1}-\left.\left(w_{, 1}+k v\right)(\bar{w}-w)\right|_{-1} ^{0}-\left.\left(w_{, 1}+k v\right)(\bar{w}-w)\right|_{0} ^{1}=0 .
\end{aligned}
$$

Чтобы получить вариационное неравенство (2.9), достаточно установить, что сумма граничных слагаемых (включая интегралы по $\gamma$ и $s_{i}$ ) в $(2.11)$ неположительна. Учитывая граничные условия (2.7) и вторую группу условий (2.4), 
достаточно доказать, что

$$
\int_{\gamma}\left[\sigma_{\nu}\left(\bar{u}_{\nu}-u_{\nu}\right)\right]+\int_{\gamma}\left[\sigma_{\tau}\left(\bar{u}_{\tau}-u_{\tau}\right)\right]-\sum_{i=1}^{2} \int_{s_{i}}\left[\sigma_{\tau}\right] p(\bar{w}-w)-\sum_{i=1}^{2} \int_{s_{i}}\left[\sigma_{\nu}\right] p(\bar{v}-v) \leq 0,
$$

т. е. с учетом краевых условий $(2.5),(2.6)$

$$
\int_{\gamma}\left[\sigma_{\nu}\left(\bar{u}_{\nu}-u_{\nu}\right)\right]-\sum_{i=1}^{2} \int_{s_{i}}\left[\sigma_{\nu}\right] p(\bar{v}-v) \leq 0 .
$$

Справедливость последнего неравенства легко проверяется с учетом краевых условий (2.6).

Предложение 1 полностью доказано.

3. Предельный переход к бесконечности по параметру жесткости. В этом разделе будет исследован предельный переход по параметру жесткости в задаче (2.8), (2.9). Будем предполагать, что жесткость одного из включений зависит от параметра $\delta>0$. В задаче $(2.8),(2.9)$ этот параметр равнялся единице. Теперь введем параметр в модель и исследуем поведение решения при $\delta \rightarrow \infty$.

Итак, рассмотрим краевую задачу вида (2.1)-(2.7) с параметром $\delta$. В этом случае все неизвестные функции будем снабжать параметром $\delta$. Требуется найти вектор перемещений $u^{\delta}=\left(u_{1}^{\delta}, u_{2}^{\delta}\right)$ и тензор напряжений $\sigma^{\delta}=\left\{\sigma_{i j}^{\delta}\right\}, i, j=1,2$, определенные в $\Omega_{\gamma}$, и перемещения точек тонкого включения $v^{\delta}, w^{\delta}$, определенные для $x_{1} \in s_{i}, i=1,2$, такие, что

$$
\begin{gathered}
-\operatorname{div} \sigma^{\delta}=f, \quad \sigma^{\delta}-B \varepsilon\left(u^{\delta}\right)=0 \quad \text { в } \Omega_{\gamma}, \\
\delta^{i-1}\left(v_{, 1111}^{\delta}+k\left(w_{, 1}^{\delta}+k v^{\delta}\right)\right)=\left[\sigma_{\nu}^{\delta}\right] p \quad \text { на } s_{i}, i=1,2, \\
\delta^{i-1}\left(-w_{, 11}^{\delta}-\left(k v^{\delta}\right)_{, 1}\right)=\left[\sigma_{\tau}^{\delta}\right] p \quad \text { на } s_{i}, i=1,2, \\
u^{\delta}=0 \text { на } \Gamma, \quad v_{, 11}^{\delta}=v_{, 111}^{\delta}=w_{, 1}^{\delta}+k v^{\delta}=0, x_{1}=-1,1, \\
{\left[u_{\nu}^{\delta}\right] \geq 0, \quad v^{\delta}=u_{\nu}^{\delta-}, \quad w^{\delta}=u_{\tau}^{\delta-} \quad \text { на } \gamma,} \\
\sigma_{\nu}^{\delta+} \leq 0, \quad \sigma_{\tau}^{\delta+}=0, \quad \sigma_{\nu}^{\delta+}\left[u_{\nu}^{\delta}\right]=0 \quad \text { на } \gamma, \\
{\left[v^{\delta}(0)\right]_{p}=\left[w^{\delta}(0)\right]_{p}=\left[v_{, 111}^{\delta}(0)\right]^{p}=\left[\left(w_{, 1}^{\delta}+k v^{\delta}\right)(0)\right]^{p}=0, \quad v_{, 11}^{\delta}(0 \pm)=0 .}
\end{gathered}
$$

Как видно, параметр жесткости равен единице для включения $\gamma_{1}$ и равен $\delta$ для включения $\gamma_{2}$. Сформулированная задача может быть записана в виде вариационного неравенства

$$
\left(u^{\delta}, v^{\delta}, w^{\delta}\right) \in K
$$

$$
\begin{aligned}
& \int_{\Omega_{\gamma}} \sigma\left(u^{\delta}\right) \varepsilon\left(\bar{u}-u^{\delta}\right)+\sum_{i=1}^{2} \int_{s_{i}} \delta^{i-1}\left(w_{, 1}^{\delta}+k v^{\delta}\right)\left(\bar{w}_{, 1}^{\delta}-w_{, 1}^{\delta}\right) \\
& +\sum_{i=1}^{2} \int_{s_{i}} \delta^{i-1}\left\{v_{, 11}^{\delta}\left(\bar{v}_{, 11}-v_{, 11}^{\delta}\right)+k\left(w_{, 1}^{\delta}+k v^{\delta}\right)\left(\bar{v}-v^{\delta}\right)\right\}-\int_{\Omega_{\gamma}} f\left(\bar{u}-u^{\delta}\right) \geq 0 \forall(\bar{u}, \bar{v}, \bar{w}) \in K \\
& (3.2)
\end{aligned}
$$


Сначала получим априорные оценки в задаче (3.1), (3.2). Из (3.1), (3.2) при $\alpha>0$ следует равенство

$$
\begin{aligned}
\int_{\Omega_{\gamma}} \sigma\left(u^{\delta}\right) \varepsilon\left(u^{\delta}\right)-\int_{\Omega_{\gamma}} f u^{\delta} & +\sum_{i=1}^{2} \int_{s_{i}} \delta^{i-1}\left(w_{, 1}^{\delta}+k v^{\delta}\right)^{2} \\
& +\sum_{i=1}^{2} \int_{s_{i}} \delta^{i-1}\left(v_{, 11}^{\delta}\right)^{2} \pm \alpha \sum_{i=1}^{2} \int_{s_{i}}\left\{\left(v^{\delta}\right)^{2}+\left(w^{\delta}\right)^{2}\right\}=0 .
\end{aligned}
$$

Выбирая малый параметр $\alpha>0$ и принимая во внимание неравенство Корна, равенства $v^{\delta}=u_{\nu}^{\delta-}, w^{\delta}=u_{\tau}^{\delta-}$ на $\gamma$ и теоремы вложения, имеем

$$
\frac{1}{2} \int_{\Omega_{\gamma}} \sigma\left(u^{\delta}\right) \varepsilon\left(u^{\delta}\right)-\sum_{i=1}^{2} \alpha \int_{s_{i}}\left\{\left(v^{\delta}\right)^{2}+\left(w^{\delta}\right)^{2}\right\} \geq c_{1}\left\|u^{\delta}\right\|_{H_{\Gamma}^{1}\left(\Omega_{\gamma}\right)^{2}}^{2}, \quad c_{1}>0 .
$$

Отсюда равномерно по $\delta$ получаем

$$
\left\|u^{\delta}\right\|_{H_{\Gamma}^{1}\left(\Omega_{\gamma}\right)^{2}}^{2} \leq c .
$$

Кроме того, для $\beta>0$ при всех $\delta \geq \delta_{0}>0, i=1,2$, справедливо неравенство

$$
\begin{aligned}
\alpha\left(v^{\delta}\right)^{2}+\alpha\left(w^{\delta}\right)^{2}+ & \delta^{i-1}\left\{\left(v_{, 11}^{\delta}\right)^{2}+\left(w_{, 1}^{\delta}\right)^{2}+k^{2}\left(v^{\delta}\right)^{2}+2 k w_{, 1}^{\delta} v^{\delta}\right\} \\
& \geq \alpha\left(v^{\delta}\right)^{2}+\alpha\left(w^{\delta}\right)^{2}+\delta_{0}^{i-1}\left\{\left(v_{, 11}^{\delta}\right)^{2}+\beta\left(w_{, 1}^{\delta}\right)^{2}-\frac{\beta k^{2}\left(v^{\delta}\right)^{2}}{1-\beta}\right\} .
\end{aligned}
$$

Поскольку при малых $\beta$ имеем $\alpha-\frac{\delta_{0}^{i-1} \beta k^{2}}{1-\beta} \geq \frac{\alpha}{2}, i=1,2$, из (3.5), (3.4), (3.3) для $\delta \geq \delta_{0}$ заключаем, что

$$
\begin{gathered}
\left\|v^{\delta}\right\|_{H^{2}\left(s_{i}\right)}^{2} \leq c, \quad\left\|w^{\delta}\right\|_{H^{1}\left(s_{i}\right)}^{2} \leq c, \quad i=1,2, \\
\delta \int_{s_{2}}\left(v_{, 11}^{\delta}\right)^{2}+\delta \int_{s_{2}}\left(w_{, 1}^{\delta}+k v^{\delta}\right)^{2} \leq c .
\end{gathered}
$$

В силу (3.5)-(3.7) можно считать, что при $\delta \rightarrow \infty$

$$
\begin{gathered}
\left(u^{\delta}, v^{\delta}, w^{\delta}\right) \rightarrow(u, v, w) \quad \text { слабо в } H_{\Gamma}^{1}\left(\Omega_{\gamma}\right)^{2} \times H^{2}\left(s_{i}\right) \times H^{1}\left(s_{i}\right), \quad i=1,2, \\
v\left(x_{1}\right)=a_{0}+a_{1} x_{1} ; \quad w_{, 1}\left(x_{1}\right)+k\left(x_{1}\right) v\left(x_{1}\right)=0, \quad x_{1} \in s_{2}, a_{0}, a_{1} \in \mathbb{R} .
\end{gathered}
$$

Введем множество допустимых перемещений для предельной задачи

$$
\begin{array}{r}
K_{r}=\left\{(u, v, w) \mid u \in H_{\Gamma}^{1}\left(\Omega_{\gamma}\right)^{2}, v \in H^{2}\left(s_{1}\right), w \in H^{1}\left(s_{1}\right),\right. \\
\left.\quad\left[u_{\nu}\right] \geq 0, v=u_{\nu}^{-}, w=u_{\tau}^{-} \text {на } \gamma,\left.\left(u_{\nu}^{-}, u_{\tau}^{-}\right)\right|_{\gamma_{2}} \in L(0,1)\right\},
\end{array}
$$

где

$L(0,1)=\left\{(v, w) \mid v\left(x_{1}\right)=b_{0}+b_{1} x_{1}, w_{, 1}\left(x_{1}\right)+k\left(x_{1}\right) v\left(x_{1}\right)=0, x_{1} \in s_{2} ; b_{0}, b_{1} \in \mathbb{R}\right\}$.

Следует отметить, что принадлежность решения множеству $K_{r}$ означает, что перемещение $u^{-}$на $\gamma_{2}$ имеет заданную структуру. А именно, перемещение $u_{\nu}^{-}=v$ должно быть непрерывной аффинной функцией, а перемещение $u_{\tau}^{-}$ должно определяться из решения обыкновенного дифференциального уравнения на интервале $s_{2}$. Это уравнение относительно $w$ в качестве правой части содержит как кривизну включения $k$, так и перемещение $v$. 
Ясно, что предельные функции $u, v, w$ из (3.8), (3.9) удовлетворяют условию $(u, v, w) \in K_{r}$. На основе (3.8), (3.9) можно перейти к пределу в (3.1), (3.2). Для этого возьмем произвольную тестовую функцию $(\bar{u}, \bar{v}, \bar{w}) \in K_{r}$. Тогда $(\bar{u}, \bar{v}, \bar{w}) \in$ $K$. После перехода к пределу при $\delta \rightarrow \infty$ в (3.1), (3.2) получим, что предельный элемент $(u, v, w)$ удовлетворяет вариационному неравенству

$$
(u, v, w) \in K_{r},
$$

$$
\begin{aligned}
\int_{\Omega_{\gamma}} \sigma(u) \varepsilon(\bar{u}-u) & -\int_{\Omega_{\gamma}} f(\bar{u}-u)+\int_{s_{1}} v_{, 11}\left(\bar{v}_{, 11}-v_{, 11}\right) \\
& +\int_{s_{1}}\left(w_{, 1}+k v\right)\left(\bar{w}_{, 1}+k \bar{v}-w_{, 1}-k v\right) \geq 0 \quad \forall(\bar{u}, \bar{v}, \bar{w}) \in K_{r} .
\end{aligned}
$$

Таким образом, доказана

Теорема 1. Решения задач (3.1), (3.2) при $\delta \rightarrow \infty$ сходятся в смысле (3.8), (3.9) к решению задачи $(3.10),(3.11)$.

Интересно выписать дифференциальную формулировку задачи (3.10), (3.11). Требуется найти вектор перемещений $u=\left(u_{1}, u_{2}\right)$ и тензор напряжений $\sigma=\left\{\sigma_{i j}\right\}, i, j=1,2$, определенные в $\Omega_{\gamma}$, и перемещения точек тонкого включения $v, w$, определенные в $s_{1} \cup s_{2}$, такие, что

$$
\begin{gathered}
-\operatorname{div} \sigma=f, \quad \sigma-B \varepsilon(u)=0 \quad \text { в } \Omega_{\gamma}, \\
v_{, 1111}+k\left(w_{, 1}+k v\right)=\left[\sigma_{\nu}\right] p, \quad-w_{, 11}-(k v)_{, 1}=\left[\sigma_{\tau}\right] p \quad \text { на } s_{1}, \\
u=0 \text { на } \Gamma ; \quad v_{, 11}=v_{, 111}=w_{, 1}+k v=0, \quad x_{1}=-1, \\
{\left[u_{\nu}\right] \geq 0, \quad \sigma_{\nu}^{+} \leq 0, \quad \sigma_{\tau}^{+}=0, \quad \sigma_{\nu}^{+}\left[u_{\nu}\right]=0 \quad \text { на } \gamma,} \\
v=u_{\nu}^{-}, w=u_{\tau}^{-} \text {на } \gamma ;\left.\quad\left(u_{\nu}^{-}, u_{\tau}^{-}\right)\right|_{\gamma_{2}} \in L(0,1), \\
{[v(0)]_{p}=[w(0)]_{p}=0, \quad v_{, 11}(0-)=0,} \\
\left.\int_{s_{2}}\left[\sigma_{\nu}\right] p \bar{v}+\int_{s_{2}}\left[\sigma_{\tau}\right] p \bar{w}+v_{, 111}(0-) \bar{v}(0)-\left(w_{, 1}+k v\right)(0-) \bar{w}(0)=0 \quad \forall(\bar{v}, \bar{w}) \in L(0,1) .13\right)
\end{gathered}
$$

Предложение 2. Задачи (3.10), (3.11) и (3.12)-(3.18) эквивалентны на классе достаточно гладких решений.

ДокАЗАТЕЛЬСтво. Предположим, что выполнено вариационное неравенство (3.10), (3.11). Сначала обычным образом докажем справедливость уравнения равновесия из (3.12). Кроме того, стандартными рассуждениями можно установить справедливость краевых условий $(3.15)$ на $\gamma$ (см. $[1,11])$. Как уже отмечалось, эти краевые условия типичны для задач такого вида. Далее подставим в $(3.11)$ в качестве тестовых функций $(\bar{u}, \bar{v}, \bar{w})=(u, v, w) \pm(\tilde{u}, \tilde{v}, \widetilde{w})$, $(\tilde{u}, \tilde{v}, \widetilde{w}) \in K_{r},\left[\tilde{u}_{\nu}\right]=0$ на $\gamma$. Будем иметь

$$
\int_{\Omega_{\gamma}} \sigma(u) \varepsilon(\tilde{u})-\int_{\Omega_{\gamma}} f \tilde{u}+\int_{s_{1}} v_{, 11} \tilde{v}_{, 11}+\int_{s_{1}}\left(w_{, 1}+k v\right)\left(\widetilde{w}_{, 1}+k \tilde{v}\right)=0 .
$$


Интегрируя по частям, находим

$$
\begin{aligned}
& -\int_{\gamma}\left[\sigma_{\nu}\right] \tilde{u}_{\nu}-\int_{\gamma}\left[\sigma_{\tau} \tilde{u}_{\tau}\right]+\int_{s_{1}} v_{, 1111} \tilde{v}-\int_{s_{1}}\left(w_{, 1}+k v\right)_{, 1} \tilde{w} \\
& \quad+\int_{s_{1}}\left(w_{, 1}+k v\right) k \tilde{v}+\left.v_{, 11} \tilde{v}_{, 1}\right|_{-1} ^{0}-\left.v_{, 111} \tilde{v}\right|_{-1} ^{0}+\left.\left(w_{, 1}+k v\right) \tilde{w}\right|_{-1} ^{0}=0 .
\end{aligned}
$$

Сначала предположим, что в (3.19) тестовые функции удовлетворяют условиям $\tilde{v}=\tilde{v}_{, 1}=\widetilde{w}=0$ при $x_{1}=-1,0-$. Получим следующее тождество, справедливое для всех выбранных функций:

$$
\begin{aligned}
-\int_{\gamma_{1}}\left[\sigma_{\nu}\right] \tilde{v}-\int_{\gamma_{1}}\left[\sigma_{\tau}\right] \widetilde{w}-\int_{\gamma_{2}}\left[\sigma_{\nu}\right] \tilde{v} & -\int_{\gamma_{2}}\left[\sigma_{\tau}\right] \widetilde{w}+\int_{s_{1}} v_{, 1111} \tilde{v} \\
& -\int_{s_{1}}\left(w_{, 1}+k v\right)_{, 1} \tilde{w}+\int_{s_{1}}\left(w_{, 1}+k v\right) k \tilde{v}=0 .
\end{aligned}
$$

Из (3.20) вытекают уравнения равновесия (3.13) и, кроме того,

$$
\int_{s_{2}}\left[\sigma_{\nu}\right] p \tilde{v}+\int_{s_{2}}\left[\sigma_{\tau}\right] p \tilde{w}=0 \quad \forall(\tilde{v}, \widetilde{w}) \in L(0,1), \tilde{v}(0)=0, \widetilde{w}(0)=0 .
$$

Вернемся к соотношению (3.19). В силу гладкости решения имеем $[v(0)]_{p}=$ $[w(0)]_{p}=0$. Поэтому получаем вторую группу краевых условий $(3.14)$ и, кроме того, $v_{, 11}(0-)=0$. Таким образом, из (3.19) следует тождество

$$
\int_{\gamma_{2}}\left[\sigma_{\nu}\right] \tilde{v}+\int_{\gamma_{2}}\left[\sigma_{\tau}\right] \widetilde{w}+v_{, 111}(0-) \tilde{v}(0)-\left(w_{, 1}+k v\right)(0-) \widetilde{w}(0)=0,
$$

справедливое для всех указанных функций $(\tilde{v}, \widetilde{w})$, что совпадает с $(3.18)$.

Отметим, что тождество (3.21) является следствием (3.18). Итак, все соотношения (3.12)-(3.18) доказаны из (3.10), (3.11).

Докажем, что из (3.12)-(3.18) следует (3.10), (3.11). Из (3.12), (3.13) для $(\bar{u}, \bar{v}, \bar{w}) \in K_{r}$ после интегрирования получим

$$
\begin{gathered}
\int_{\gamma}[\sigma \nu(\bar{u}-u)]+\int_{\Omega_{\gamma}} \sigma(u) \varepsilon(\bar{u}-u)-\int_{\Omega_{\gamma}} f(\bar{u}-u) \\
+\int_{s_{1}} v_{, 11}\left(\tilde{v}_{, 11}-v_{, 11}\right)+\int_{s_{1}}\left(w_{, 1}+k v\right)\left(\bar{w}_{, 1}+k \bar{v}-w_{, 1}-k v\right) \\
-\int_{s_{1}}\left[\sigma_{\tau}\right] p(\bar{w}-w)-\int_{s_{1}}\left[\sigma_{\nu}\right] p(\bar{v}-v) \\
-\left.v_{, 11}\left(\bar{v}_{, 1}-v_{, 1}\right)\right|_{-1} ^{0}+\left.v_{, 111}(\bar{v}-v)\right|_{-1} ^{0}-\left.\left(w_{, 1}+k v\right)(\bar{w}-w)\right|_{-1} ^{0}=0 .
\end{gathered}
$$

Для того чтобы получить вариационное неравенство (3.11) из (3.22), достаточно доказать, что

$$
\int_{\gamma_{1}}\left[\sigma_{\nu}\left(\bar{u}_{\nu}-u_{\nu}\right)\right]+\int_{\gamma_{1}}\left[\sigma_{\tau}\left(\bar{u}_{\tau}-u_{\tau}\right)\right]
$$




$$
\begin{aligned}
+\int_{\gamma_{2}}\left[\sigma_{\nu}\left(\bar{u}_{\nu}-u_{\nu}\right)\right]+\int_{\gamma_{2}}\left[\sigma_{\tau}\left(\bar{u}_{\tau}-u_{\tau}\right)\right]-\int_{s_{1}}\left[\sigma_{\tau}\right] p(\bar{w}-w)-\int_{s_{1}}\left[\sigma_{\nu}\right] p(\bar{v}-v) \\
+\left(v_{, 111}(\bar{v}-v)\right)(0-)-\left(\left(w_{, 1}+k v\right)(\bar{w}-w)\right)(0-) \leq 0 .
\end{aligned}
$$

Справедливость последнего неравенства нетрудно проверяется с учетом краевых условий (3.15), (3.16), (3.18).

Предложение 2 доказано.

Таким образом, дифференциальная формулировка задачи (3.10), (3.11) в виде (3.12)-(3.18) наряду с условиями сопряжения (3.17) содержит тождество (3.18). Это тождество включает как нелокальное слагаемое (интегралы по $s_{2}$ ), так и значения искомых функций в точке $0-$.

Полученные результаты будут справедливы и в случае, если на берегах трещины заданы линейные краевые условия. Приведем формулировки соответствующих краевых задач. Задача (2.1)-(2.7) будет трансформирована в следующую. Найти вектор перемещений $u=\left(u_{1}, u_{2}\right)$ и тензор напряжений $\sigma=\left\{\sigma_{i j}\right\}$, $i, j=1,2$, определенные в $\Omega_{\gamma}$, и перемещения точек тонких включений $v, w$, определенные в $s_{1} \cup s_{2}$, такие, что

$$
\begin{gathered}
-\operatorname{div} \sigma=f, \quad \sigma-B \varepsilon(u)=0 \quad \text { в } \Omega_{\gamma}, \\
v_{, 1111}+k\left(w_{, 1}+k v\right)=\left[\sigma_{\nu}\right] p \text { на } s_{i}, \quad i=1,2, \\
-w_{, 11}-(k v)_{, 1}=\left[\sigma_{\tau}\right] p \text { на } s_{i}, \quad i=1,2, \\
u=0 \text { на } \Gamma ; \quad v_{, 11}=v_{, 111}=w_{, 1}+k v=0, \quad x_{1}=-1,1, \\
\sigma^{+} \nu=0, \quad v=u_{\nu}^{-}, \quad w=u_{\tau}^{-} \quad \text { на } \gamma \\
{[v(0)]_{p}=[w(0)]_{p}=\left[v_{, 111}(0)\right]^{p}=\left[\left(w_{, 1}+k v\right)(0)\right]^{p}=0, \quad v_{, 11}(0 \pm)=0 .}
\end{gathered}
$$

Предельная краевая задача сопряжения упругого и жесткого тонких включений (3.12)-(3.18) также будет трансформирована в случае линейных краевых условий на берегах трещин. Формулировка приобретает следующий вид. Найти вектор перемещений $u=\left(u_{1}, u_{2}\right)$ и тензор напряжений $\sigma=\left\{\sigma_{i j}\right\}, i, j=1,2$, определенные в $\Omega_{\gamma}$, и перемещения точек тонкого включения $v, w$, определенные в $s_{1} \cup s_{2}$, такие, что

$$
\begin{gathered}
-\operatorname{div} \sigma=f, \quad \sigma-B \varepsilon(u)=0 \quad \text { в } \Omega_{\gamma}, \\
v_{, 1111}+k(w, 1+k v)=\left[\sigma_{\nu}\right] p,-w_{, 11}-(k v)_{, 1}=\left[\sigma_{\tau}\right] p \quad \text { на } s_{1}, \\
u=0 \text { на } \Gamma ; \quad v_{, 11}=v_{, 111}=w_{, 1}+k v=0, \quad x_{1}=-1, \\
\sigma^{+} \nu=0, \quad v=u_{\nu}^{-}, \quad w=u_{\tau}^{-} \quad \text { на } \gamma ;\left.\quad\left(u_{\nu}^{-}, u_{\tau}^{-}\right)\right|_{\gamma_{2}} \in L(0,1), \\
{[v(0)]_{p}=[w(0)]_{p}=0, \quad v_{, 11}(0-)=0,} \\
\int_{s_{2}}\left[\sigma_{\nu}\right] p \bar{v}+\int_{s_{2}}\left[\sigma_{\tau}\right] p \bar{w}+v_{, 111}(0-) \bar{v}(0)-\left(w_{, 1}+k v\right)(0-) \bar{w}(0)=0 \quad \forall(\bar{v}, \bar{w}) \in L(0,1) .
\end{gathered}
$$

4. Задача равновесия. Случай без излома. В этом разделе приведем результаты анализа задачи равновесия упругого тела с двумя слабо искривленными включениями $\gamma_{1}, \gamma_{2}$ для случая, когда излом в точке $(0,0)$ отсутствует. Отсутствие излома в точке $(0,0)$ означает, что включения $\gamma_{1}, \gamma_{2}$ связаны между собой. Фактически имеется одно включение $\gamma$ с возможным нарушением гладкости срединной линии в точке $(0,0)$. Поскольку гладкость функции $\varphi$ может 
нарушаться в точке 0 , так же, как в разд. 2, уравнения равновесия для $\gamma_{1}$ и $\gamma_{1}$ следует выписывать отдельно. Таким образом, по-прежнему будем рассматривать два включения $\gamma_{1}, \gamma_{2}$, а в точке $(0,0)$ следует формулировать условия сопряжения. Одним из этих условий будет сохранение угла между включениями в процессе деформирования. Как и ранее, для простоты будем считать, что $\varphi, 1(0+)=0$.

Формулировка соответствующей задачи равновесия состоит в следующем. Требуется найти вектор перемещений $u=\left(u_{1}, u_{2}\right)$ и тензор напряжений $\sigma=$ $\left\{\sigma_{i j}\right\}, i, j=1,2$, определенные в $\Omega_{\gamma}$, и перемещения точек тонких включений $v, w$, определенные в $s_{1} \cup s_{2}$, такие, что

$$
\begin{gathered}
-\operatorname{div} \sigma=f, \quad \sigma-B \varepsilon(u)=0 \quad \text { в } \Omega_{\gamma}, \\
v_{, 1111}+k\left(w_{, 1}+k v\right)=\left[\sigma_{\nu}\right] p,-w_{, 11}-(k v)_{, 1}=\left[\sigma_{\tau}\right] p \text { на } s_{i}, \quad i=1,2, \\
u=0 \text { на } \Gamma ; \quad v_{, 11}=v_{, 111}=w_{, 1}+k v=0, \quad x_{1}=-1,1, \\
{\left[u_{\nu}\right] \geq 0, \quad v=u_{\nu}^{-}, \quad w=u_{\tau}^{-}, \quad \sigma_{\nu}^{+} \leq 0, \quad \sigma_{\tau}^{+}=0, \quad \sigma_{\nu}^{+}\left[u_{\nu}\right]=0 \text { на } \gamma} \\
{[v(0)]_{p}=[w(0)]_{p}=\left[v_{, 1}(0)\right]=0,} \\
{\left[v_{, 11}(0)\right]=\left[v_{, 111}(0)\right]^{p}=\left[\left(w_{, 1}+k v\right)(0)\right]^{p}=0}
\end{gathered}
$$

где $[m(0)]=m(0+)-m(0-)$. Как видно, наряду с сохранением угла между включениями в точке сопряжения скачок момента также нулевой в точке $(0,0)$. Задача (4.1)-(4.6) допускает вариационную формулировку. Введем множество допустимых перемещений

$$
\begin{aligned}
K^{0}=\left\{(u, v, w) \mid u \in H_{\Gamma}^{1}\left(\Omega_{\gamma}\right)^{2}, v \in H^{2}\left(s_{i}\right), w\right. & \in H^{1}\left(s_{i}\right), i=1,2, \\
& {\left.\left[u_{\nu}\right] \geq 0, v=u_{\nu}^{-}, w=u_{\tau}^{-} \text {на } \gamma,\left[v_{, 1}(0)\right]=0\right\} . }
\end{aligned}
$$

Как и ранее, функционал энергии имеет вид

$$
\pi(u, v, w)=\frac{1}{2} \int_{\Omega_{\gamma}} \sigma(u) \varepsilon(u)-\int_{\Omega_{\gamma}} f u+\frac{1}{2} \sum_{i=1}^{2} \int_{s_{i}} v_{, 11}^{2}+\frac{1}{2} \sum_{i=1}^{2} \int_{s_{i}}\left(w_{, 1}+k v\right)^{2},
$$

а задача минимизации:

$$
\text { найти }(u, v, w) \in K^{0} \text { так, что } \pi(u, v, w)=\inf _{K^{0}} \pi,
$$

имеет единственное решение, удовлетворяющее вариационному неравенству

$$
\begin{gathered}
(u, v, w) \in K^{0}, \\
\int_{\Omega_{\gamma}} \sigma(u) \varepsilon(\bar{u}-u)-\int_{\Omega_{\gamma}} f(\bar{u}-u)+\sum_{i=1}^{2} \int_{s_{i}} v_{, 11}\left(\bar{v}_{, 11}-v_{, 11}\right) \\
+\sum_{i=1}^{2} \int_{s_{i}}\left\{\left(w_{, 1}+k v\right)\left(\bar{w}_{, 1}+k \bar{v}-w_{, 1}-k v\right)\right\} \geq 0 \quad \forall(\bar{u}, \bar{v}, \bar{w}) \in K^{0} .
\end{gathered}
$$

Можно проверить, что на классе гладких решений задачи (4.7), (4.8) и (4.1)-(4.6) эквиваленты в том смысле, что (4.7), (4.8) можно получить из (4.1)(4.6) и, наоборот, все соотношения (4.1)-(4.6) вытекают из (4.7), (4.8). 
Как в разд. 3, можно ввести параметр $\delta>0$ в модель (4.1)-(4.6) и исследовать предельный переход при стремлении этого параметра к бесконечности, при этом жесткость включения $\gamma_{1}$ будет фиксирована и равна единице, а жесткость включения $\gamma_{2}$ будет равна $\delta$. Рассмотрим вариационное неравенство

$$
\begin{gathered}
\left(u^{\delta}, v^{\delta}, w^{\delta}\right) \in K^{0} \\
\int_{\Omega_{\gamma}} \sigma\left(u^{\delta}\right) \varepsilon\left(\bar{u}-u^{\delta}\right)+\sum_{i=1}^{2} \int_{s_{i}} \delta^{i-1} v_{, 11}^{\delta}\left(\bar{v}_{, 11}-v_{, 11}^{\delta}\right) \\
+\sum_{i=1}^{2} \int_{s_{i}} \delta^{i-1}\left\{\left(w_{, 1}^{\delta}+k v^{\delta}\right)\left(\bar{w}_{, 1}+k \bar{v}-w_{, 1}^{\delta}-k v^{\delta} \quad r\right)\right\}-\int_{\Omega_{\gamma}} f\left(\bar{u}-u^{\delta}\right) \geq 0 \forall(\bar{u}, \bar{v}, \bar{w}) \in K^{0}
\end{gathered}
$$

Как оказывается, при $\delta \rightarrow \infty$ включение $\gamma_{2}$ становится жестким, т. е. поле перемещений для этого включения будет иметь заданную структуру.

Чтобы сформулировать предельную задачу, соответствующую $\delta \rightarrow \infty$, введем множество допустимых перемещений

$$
\begin{aligned}
& K_{r}^{0}=\left\{(u, v, w) \mid u \in H_{\Gamma}^{1}\left(\Omega_{\gamma}\right)^{2}, v \in H^{2}\left(s_{1}\right), w \in H^{1}\left(s_{1}\right),\right. \\
& \left.\quad\left[u_{\nu}\right] \geq 0, v=u_{\nu}^{-}, w=u_{\tau}^{-} \text {на } \gamma,\left.\left(u_{\nu}^{-}, u_{\tau}^{-}\right)\right|_{\gamma_{2}} \in L(0,1),\left[v_{, 1}(0)\right]=0\right\} .
\end{aligned}
$$

Множество $L(0,1)$ определяется так же, как в разд. 3. Не приводя подробностей, отметим, что из последовательности решений задач (4.9), (4.10) можно выбрать подпоследовательность такую, что при $\delta \rightarrow \infty$

$$
\begin{gathered}
\left(u^{\delta}, v^{\delta}, w^{\delta}\right) \rightarrow(u, v, w) \quad \text { слабо в } H_{\Gamma}^{1}\left(\Omega_{\gamma}\right)^{2} \times H^{2}\left(s_{i}\right) \times H^{1}\left(s_{i}\right), \quad i=1,2, \\
v\left(x_{1}\right)=a_{0}+a_{1} x_{1} ; \quad w_{, 1}\left(x_{1}\right)+k\left(x_{1}\right) v\left(x_{1}\right)=0, \quad x_{1} \in s_{2} ; a_{0}, a_{1} \in \mathbb{R} .
\end{gathered}
$$

При этом предельные функции $u, v, w$ будут удовлетворять вариационному неравенству

$$
\begin{gathered}
(u, v, w) \in K_{r}^{0}, \\
\int_{\Omega_{\gamma}} \sigma(u) \varepsilon(\bar{u}-u)-\int_{\Omega_{\gamma}} f(\bar{u}-u)+\int_{s_{1}} v_{, 11}\left(\bar{v}_{, 11}-v_{, 11}\right) \\
+\int_{s_{1}}\left(w_{, 1}+k v\right)\left(\bar{w}_{, 1}+k \bar{v}-w_{, 1}-k v\right) \geq 0 \quad \forall(\bar{u}, \bar{v}, \bar{w}) \in K_{r}^{0} .
\end{gathered}
$$

Соответствующий результат сформулируем в виде теоремы.

Теорема 2. Решения задач (4.9), (4.10) при $\delta \rightarrow \infty$ сходятся в смысле (4.11), (4.12) к решению задачи (4.13), (4.14).

Наконец, приведем дифференциальную формулировку задачи (4.13), (4.14). Требуется найти вектор перемещений $u=\left(u_{1}, u_{2}\right)$ и тензор напряжений $\sigma=$ $\left\{\sigma_{i j}\right\}, i, j=1,2$, определенные в $\Omega_{\gamma}$, и перемещения точек тонкого включения $v, w$, определенные в $s_{1} \cup s_{2}$, такие, что

$$
\begin{gathered}
-\operatorname{div} \sigma=f, \quad \sigma-B \varepsilon(u)=0 \quad \text { в } \Omega_{\gamma}, \\
v_{, 1111}+k\left(w_{, 1}+k v\right)=\left[\sigma_{\nu}\right] p, \quad-w_{, 11}-(k v)_{, 1}=\left[\sigma_{\tau}\right] p \quad \text { на } s_{1}, \\
u=0 \text { на } \Gamma ; \quad v_{, 11}=v_{, 111}=w_{, 1}+k v=0, \quad x_{1}=-1,
\end{gathered}
$$




$$
\begin{gathered}
{\left[u_{\nu}\right] \geq 0, \quad \sigma_{\nu}^{+} \leq 0, \quad \sigma_{\tau}^{+}=0, \quad \sigma_{\nu}^{+}\left[u_{\nu}\right]=0 \text { на } \gamma,} \\
v=u_{\nu}^{-}, w=u_{\tau}^{-} \text {на } \gamma ;\left.\quad\left(u_{\nu}^{-}, u_{\tau}^{-}\right)\right|_{\gamma_{2}} \in L(0,1), \\
{[v(0)]_{p}=[w(0)]_{p}=\left[v_{, 1}(0)\right]=0,} \\
\int_{s_{2}}\left[\sigma_{\nu}\right] p \bar{v}+\int_{s_{2}}\left[\sigma_{\tau}\right] p \bar{w}+v_{, 111}(0-) \bar{v}(0) \\
-v_{, 11}(0-) \bar{v}_{, 1}(0)-\left(w_{, 1}+k v\right)(0-) \bar{w}(0)=0 \quad \forall(\bar{v}, \bar{w}) \in L(0,1) .
\end{gathered}
$$

Как видно, по сравнению с соответствующим краевым условием (3.18) при формулировке задачи равновесия с изломом между включениями нелокальное краевое условие (4.21) содержит вторую производную слева от функции $v$ в точке 0.

На классе гладких решений задачи (4.13), (4.14) и (4.15)-(4.21) эквивалентны. Детали доказательства опустим, так как они в целом аналогичны приведенным в разд. 3 .

Так же, как в конце разд. 3 , в рассматриваемом в разд. 4 случае отсутствия излома в точке $(0,0)$ можно сформулировать задачу сопряжения двух тонких упругих слабо искривленных включений, а также задачу сопряжения упругого и жесткого включений для случая линейных краевых условий на берегах трещины. Подробности приводить не будем.

Подчеркнем, что все полученные в данной работе результаты установлены для нелинейных моделей, не допускающих взаимного проникания противоположных берегов трещин. В то же время аналогичные результаты будут справедливы и в более простом случае классических линейных краевых условий на берегах трещин.

\section{ЛИТЕРАТУРА}

1. Хлуднев А. М. Задачи теории упругости в негладких областях. М.: Физматлит, 2010.

2. Khludnev A. M., Kovtunenko V. A. Analysis of cracks in solids. Southampton; Boston: WIT Press, 2000.

3. Kovtunenko V. A., Leugering G. A shape-topological control problem for nonlinear crackdefect interaction: the anti-plane variational model // SIAM J. Control Optim. 2016. V. 54, N 3. P. 1329-1351.

4. Knees D., Mielke A. Energy release rate for cracks in finite-strain elasticity // Math. Methods Appl. Sci. 2008. V. 31, N 5. P. 501-518.

5. Knees D., Schroder A. Global spatial regularity for elasticity models with cracks, contact and other nonsmooth constraints // Math. Methods Appl. Sci. 2012. V. 35, N 15. P. 1859-1884.

6. Lazarev N. P., Rudoy E. M. Shape sensitivity analysis of Timoshenko's plate with a crack under the nonpenetration condition // Z. Angew. Math. Mech. 2014. V. 94, N 9. P. 730-739.

7. Рудой Е. М. Асимптотика функционала энергии для смешанной краевой задачи четвертого порядка в области с разрезом // Сиб. мат. журн. 2009. Т. 50, № 2. С. 430-445.

8. Khludnev A. M., Leugering G. R. On Timoshenko thin elastic inclusions inside elastic bodies // Math. Mech. Solids. 2015. V. 20, N 5. P. 495-511.

9. Рудой Е. М. Асимптотика функционала энергии для трехмерного тела с жестким включением и трещиной // Прикл. механика и техн. физика. 2011. Т. 52, № 2. С. 114-127.

10. Rudoy E. M. Shape derivative of the energy functional in a problem for a thin rigid inclusion in an elastic body // Z. Angew. Math. Phys. 2015. V. 66, N 4. P. 1923-1937.

11. Хлуднев A. М. Слабо искривленное включение в упругом теле при наличии отслоения // Изв. РАН. Механика твердого тела. 2015. № 5. С. 131-144.

12. Щербаков В. В. Об одной задаче управления формой тонких включений в упругих телах // Сиб. журн. индустр. математики. 2013. Т. 16, № 1. С. 138-147.

13. Щербаков В. В. О выборе оптимальной формы тонких жестких включений в упругих телах // Прикл. механика и техн. физика. 2015. Т. 56, № 2. С. 178-187. 
14. Lazarev N. P. Shape sensitivity analysis of the energy integrals for the Timoshenko-type plate containing a crack on the boundary of a rigid inclusion // Z. Angew. Math. Phys. 2015. V. 66, N 4. P. 2025-2040.

15. Le Dret H. Modeling of the junction between two rods // J. Math. Pures Appl. 1989. V. 68. P. 365-397.

16. Le Dret H. Modeling of a folded plate // Computational Mechanics. 1990. V. 5. P. 401-416.

17. Ciarlet P. G., Le Dret H., Nzengwa R. Junctions between three dimensional and two dimensional linearly elastic structures // J. Math. Pures Appl. 1989. V. 68. P. 261-295.

18. Titeux I., Sanchez-Palencia E. Junction of thin plates // Europ. J. Mech. A/Solids. 2000. V. 19, N 3. P. 377-400.

19. Gaudiello A., Zappale E. Junction in a thin multidomain for a fourth order problem // Math. Models Methods Appl. Sci. 2006. V. 16. P. 1887-1918.

20. Gaudiello A., Monneau R., Mossino J., Mura F., Sili A. Junction of elastic plates and beams // ESAIM Control Optim. Calc. Var. 2007. V. 13. P. 419-457.

21. Неустроева Н. В., Лазарев Н. П. Задача сопряжения для упругих балок Бернулли Эйлера и Тимошенко // Сиб. электрон. мат. изв. 2016. Т. 13. С. $26-37$.

22. Боган Ю. А. Об условиях сопряжения А. А. Самарского и В. Б. Андреева в теории упругих балок // Мат. заметки. 2012. Т. 92, № 5. С. 661-669.

23. Khludnev A. M., Faella L., Popova T. S. Junction problem for rigid and Timoshenko elastic inclusions in elastic bodies // Math. Mech. Solids. 2017. V. 22, N 4. P. 737-750.

24. Faella L., Khludnev A. M. Junction problem for elastic and rigid inclusions in elastic bodies // Math. Methods Appl. Sci. 2016. V. 39, N 12. P. 3381-3390.

25. Khludnev A. M., Popova T. S. Junction problem for rigid and semi-rigid inclusions in elastic bodies // Arch. Appl. Mech. 2016. V. 86, N 9. P. 1565-1577.

26. Khludnev A. M., Popova T. S. Junction problem for Euler-Bernoulli and Timoshenko elastic inclusions in elastic bodies // Quart. Appl. Math. 2016. V. 74, N 4. P. 705-718.

27. Khludnev A. M., Popova T. S. On the mechanical interplay between Timoshenko and semirigid inclusions embedded in elastic bodies // Z. Angew. Math. Mech. 2017. V. 97, N 11. P. 1406-1417.

28. Khludnev A. M., Popova T. S. Semirigid inclusions in elastic bodies: Mechanical interplay and optimal control // Comp. Math. Appl. 2019. V. 77, N 1. P. 253-262.

29. Вольмир А. С. Нелинейная динамика пластинок и оболочек. М.: Наука, 1972.

Поступила в редакиию 4 декабря 2019 г.

После доработки 17 декабря 2019 г.

Принята к публикации 25 декабря 2019 г.

Хлуднев Александр Михайлович

Математический центр в Академгородке,

Институт математики им. С. Л. Соболева СО РАН,

пр. Академика Коптюга, 4, Новосибирск 630090;

Институт гидродинамики им. М. А. Лаврентьева СО РАН,

пр. Лаврентьева, 15, Новосибирск 630090

khlud@hydro.nsc.ru

Попова Татьяна Семеновна

Северо-Восточный федеральный университет им. М. К. Аммосова,

ул. Белинского, 58, Якутск 677000

ptsokt@mail.ru 